\title{
THREAD ROLLING REPAIR METHOD FOR 3D PRINTED BOLTS
}

\author{
Katzwinkel, Tim (1); \\ Mohammad, Esanula (1); \\ Silberkuhl, Philipp (2); \\ Löwer, Manuel (3) \\ 1: Institute for Product-Innovations, University of Wuppertal; \\ 2: Silbertool GmbH; \\ 3: Product Safety and Quality Engineering, University of Wuppertal
}

\begin{abstract}
Within this paper, a new method for the quality refinement of external metric standard threads on 3Dprinted bolts is presented. The repair method is based on the application of thread rolling technology, which is applied in terms of cold forming after the regular printing has been finished. The explorative study proves, that the investigated technology has a good potential to solve known precision issues in FDM 3D printing regarding the required accuracy for function fulfilling standardized threads. The application of thread rolling can be done manually and with minimal tool effort, which makes the technology particularly attractive for low cost applications.
\end{abstract}

Keywords: Design for Additive Manufacturing (DfAM), Optimisation, Sustainability

\section{Contact:}

Katzwinkel, Tim

BUW University of Wuppertal

Institute for Product-Innovations

Germany

tim.katzwinkel@rwth-aachen.de 


\section{INTRODUCTION}

In the past few years, 3D printing has emerged from a niche technology to a broad worldwide creativity tool used by enthusiasts and hobbyists as well as professional product designers or artists. Since the technological beginnings in the 1980s, the systems for generative manufacturing have been made available to a mass market by means of low-cost components and modifiable setups, whereby 3D printing technology has achieved unprecedented popularity since 2010 (Horvath and Cameron, 2014, p. 3ff). By addressing the broad community of makers inside and outside of the traditional company context, the exchange and modification of geometric 3D-CAD shapes has evolved enormously. Today, internet platforms for the exchange of precise 3D geometric models as well as special solutions for 3Dprinted parts and products have evolved in a ever-rising number. For example, the internet platform "thingiverse", that has been founded by a manufacturer of 3D-printers in 2008, claims to have more than $3,965,950$ registered community members and about 1,950,250 uploaded designs (Thingiverse, 2020).

One of the most common additive manufacturing technology used in desktop 3d printers is the so called fused-deposition-modelling (short: FDM) (Sculpteo, 2020, p. 9). To produce a threedimensional object with FDM technology, plastic filament is melted to a certain viscosity and is then applied to the printing surface (so called „build-plate") by means of single two-dimensional layers, which are stacked upon each other stepwise (Horvath and Cameron, 2014, p. 11ff).

Although this technology brings many advantages in terms of rapid prototyping, 3D printing on the other hand poses a major problem in terms of misprints and resulting waste. According to an online survey from early 2019 , the majority of $3 \mathrm{~d}$ printer users consume approximately $24 \mathrm{~kg}$ of filament annually (Toor 2019). Here, more than 70 percent of respondents reported a waste percentage of more than $6 \%$ up to even $40 \%$. According to the study, reducing an average of only $10 \%$ of waste could have saved up to 8 million kilograms of plastic filament in 2020 .

Due to process parameters (e.g. filament, temperature, printing speed, environment) and specific machine tolerances, FDM print results can not be produced with homogeneously quality throughout all printer models and brands compared to nominal tolerances of common machine elements. Consequently, printing a metric thread on different printers results in various deviations compared to the nominal standard geometry, e.g. the thread profile. As a result, printing a standard metric bolt on different machines does not always allow the application of a suiting nominal stock nut according to ISO standards. Therefore, users are in need of methods to compensate the machine and environment related deviations.

\section{STATE OF THE ART}

To date, various techniques have been investigated to deal with defects caused by poor filament adhesion, poor ambient conditions and production-related inaccuracies in the print result. One possibility for increasing strength is, for example, the thermal post-treatment of the printed products. It has been proven that the strength of the components can be significantly increased depending on temperature, exposure time and layer height (Kuznetsov et al., 2019).

Although temperature post-treatment has a generally positive effect on the strength of a component, it cannot influence the dimensional accuracy of the printed geometry. Therefore, other techniques for quality assurance have been established in FDM printing.

An important, although obvious, method is the share of the exact device-specific printing parameters and configuration settings beforehand. This method is practiced, for example, within the thingiverse portal on the basis of a broad community, so that specific parts come with several setting variants for the most common desktop printers may even be visible there (Thingiverse, 2020). However, the platform's basic focus on desktop 3D printers and the maker scene, as well as the volunteer basis for providing information, are disadvantages in terms of a reliable quality optimization. As a result, not all designs and devices always have corresponding print parameters available, which is why this method is effective but not suitable for all applications.

Another current technological development is the AI-supported prediction of possible deviations based on continuous measurement of the printing process using sensors (Systems, 2020). This promising technology is currently still in a development phase and therefore not suitable for productive use. Furthermore, a device-specific usability is expected, which is why the approach cannot be evaluated as generically suitable for any FDM application case. Finally, AI technology at the current state can detect misprints and stop the printers for saving time - but it does not help improving accuracy of the process. 
In the field of classical post-processing of FDM printed components, machining processes are in principle suitable as well - if their application is indicated in the context of the plastic used (e.g. melting temperature as a limit). A common method for thread optimization in FDM printing is the cutting of threads after printing with the help of a threading die. Unfortunately, during this process material is removed, which affects the load capacity of the printed object.

In the context of functional and standardized component geometries, such as standard threads, dimensional accuracy is particularly important when using other standardized components in rapid prototyping of product assemblies. When printing a metric standard thread in example, the problem of quality is particularly evident in FDM printing. While 3D CAD systems nowadays support the automated three-dimensional modelling of standard threads across the board, the printed components often cannot be combined with a metric nut thread without further processing. This is of particular relevance wherever machine elements have to be combined with FDM printed components. Since standard parts (e.g. nuts, sleeves) are a safe and cost-efficient alternative to self-designed FDM components and also allow for more extensive material combinations, the rapid generation of metric standard threads is an important problem in the field of FDM-based rapid prototyping.

\subsection{Problem statement and justification of research}

The techniques described so far clearly show that there is a need for action in the form of a reliable and easy-to-use method (low-tech) for thread repair in FDM printing that can be used independently of the equipment. Therefore, the application of a previously unused repair technique is presented below and evaluated in an exploratory experimental study. As an example of application, the thread form for the use of standard nuts is guaranteed to conform to standards.

The technique presented in this paper has never been applied to FDM Materials or 3D-printed parts before, which results in absolute novelty and innovation of the presented solution approach. To the same extent, the load studies and results of the process comparisons in this paper reveal new findings in the context of FDM thread repairing techniques.

\section{THREAD ROLLING AS ALTERNATIVE REPAIR TECHNOLOGY}

Thread rolling is one of the profile cross rolling processes and has the advantage that rolled threads have higher strength values compared to machined threads. Until today, the cold forming of threads has only been used in industrial series production for minimum lot sizes of several thousand parts (Klocke 2013, S. 200). But with the help of a special innovative tool development from Germany by Silbertool $\mathrm{GmbH}$ in 2010, it is nowadays possible to repair damaged threads manually using the thread rolling process. This technology, which has been patented worldwide since then, enables thread rolling to be used within a manual hand tool. At the time of writing, no comparable tool with the same principle of rolling threads is existing worldwide. Therefore, this specific product and its technique is considered unique and fundamentally new at the current state of the art regarding manual cold forming of thread geometries in general.

The main advantages of thread rolling in comparison to thread re-cutting are the material forming, which means no material loss and an increase in hardness due to work hardening. Further advantages are press smooth surfaces, an optimal fiber flow and a reduction of notch sensitivity. (Silbertool, 2020a)

\subsection{Basic principles of manual thread rolling}

Industrial thread rolling is a very old and very proven technology. The advantage of the manual rolling method presented here is that it allows manual thread rolling on site. At the beginning the pitch of the thread to be repaired must be determined. Then the profile rolls of the tool with the corresponding pitch can be used. The thread to be repaired is inserted between the profile roller and the counter roller and the distance between the rollers can be changed using a hexagon socket. In Figure 1 the tool and the required direction of movement are shown. The distance between profile roller and counter rollers can be adjusted by means of an adjusting screw. This mechanism allows the workpiece to be fixed in one place. After the object to be repaired has been firmly fixed, the tool can now be turned on its own axis by hand force and the damaged thread is rolled into the correct shape. 

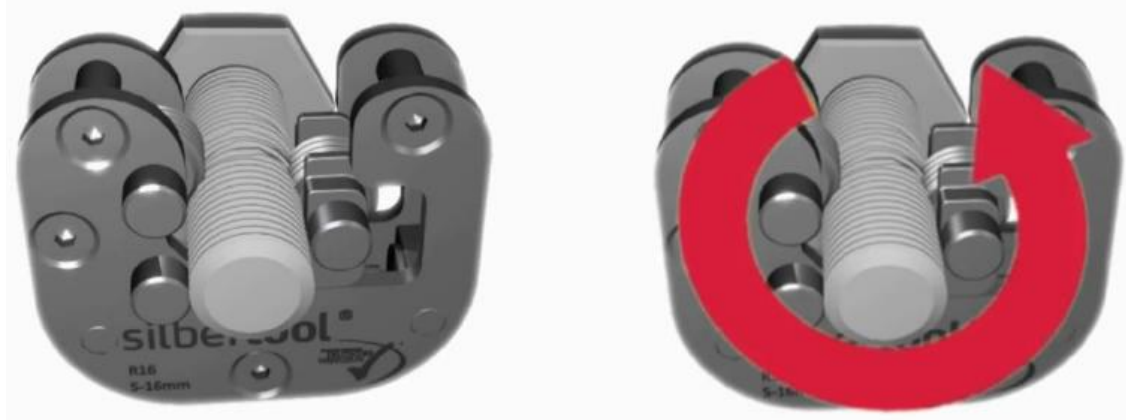

Figure 1: Silbertool thread-saver tool R16X and application direction (Silbertool, 2020b)

In the following chapter, the advantage of rolled threads in the context of metal applications is explained, which also might have a positive effect on the application in FDM printing.

\subsection{Properties of rolled threads in metal applications}

During the forming of the material, the material solidifies, which is based on complex plastomechanical processes in the crystal structure. This hardening is measurable and can be determined by a hardness measurement. Figure 2 shows the hardness curve on the rolled thread. (VDI, 1989)

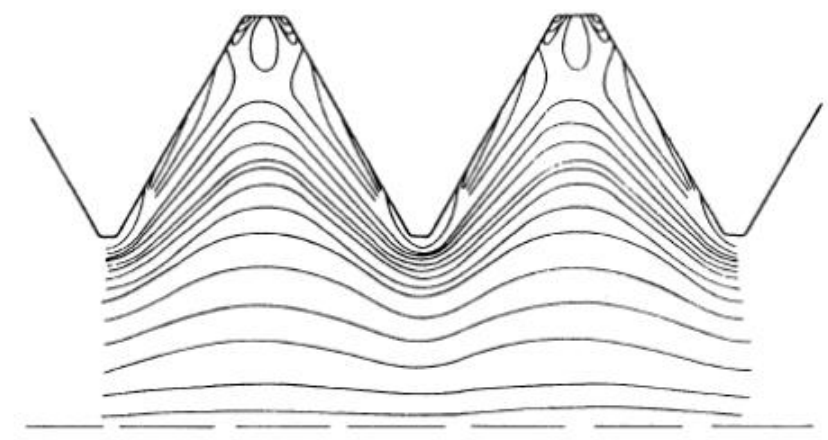

Figure 2: Hardness curve on the rolled thread (VDI, 1989)

Hardening at the rolled thread leads to a local increase in the mechanical load capacity of the material. In measuring tests, Kinzel was able to prove that a breakage failure of repaired threaded bolts using the rolling method showed approximately the same breakage behaviour as the undamaged samples. In both cases, the minimum breaking load required by ISO 898-1 was exceeded. (Kinzel, 2015)

\section{ADOPTING ROLLED TREAD REPAIR TECHNOLOGY IN FDM PRINTING}

With the possibility of a manual, locally applicable thread rolling of external threads according to the manual rolling method for diameter ranges from $5 \mathrm{~mm}$ to $110 \mathrm{~mm}$, an attractive alternative arises for the problem of iterative optimized thread generation or thread cutting in the field of rapid prototyping with FDM technology. In this chapter, the experimental exploration of this new application case is discussed in detail.

\subsection{Prerequisites and manual application}

In order to test the basic suitability of the method, a reference component in the form of a M10x40 screw with metric standard thread and hexagon socket was first produced on an FMD desktop printer of type i3Mega from Anycubic with $100 \%$ Infill settings. This unintentionally revealed the problem of quality deviations in the form of a non-standard thread pitch as described in chapter 2 . By using the manual rolling technology, the defect could be corrected afterwards. Figure 3 shows the comparison of the thread pitches before and after the thread rolling application. In addition to the more precise pitch, the light discolorations in the repaired thread can be identified, which were caused by slight material abrasion. The loss of material during thread rolling, measured using a laboratory balance, is approx. $0.3 \%$. 


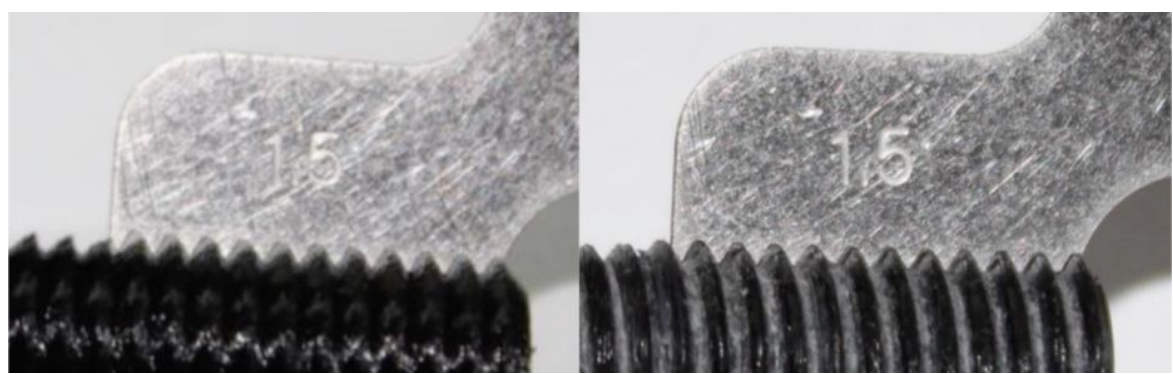

Figure 3: Comparison of the thread pitch of the original printing part (left) and re-rolled thread (right)

As the microscopic images below show, the individual layers of the FDM component were significantly flattened during the forming process. The mechanical formation of the standardized thread profile and the abrasion in the thread base can be seen as well. Chapter 4.2 investigates experimentally how these changes in the surface condition of the threads affect the load carrying capacity.
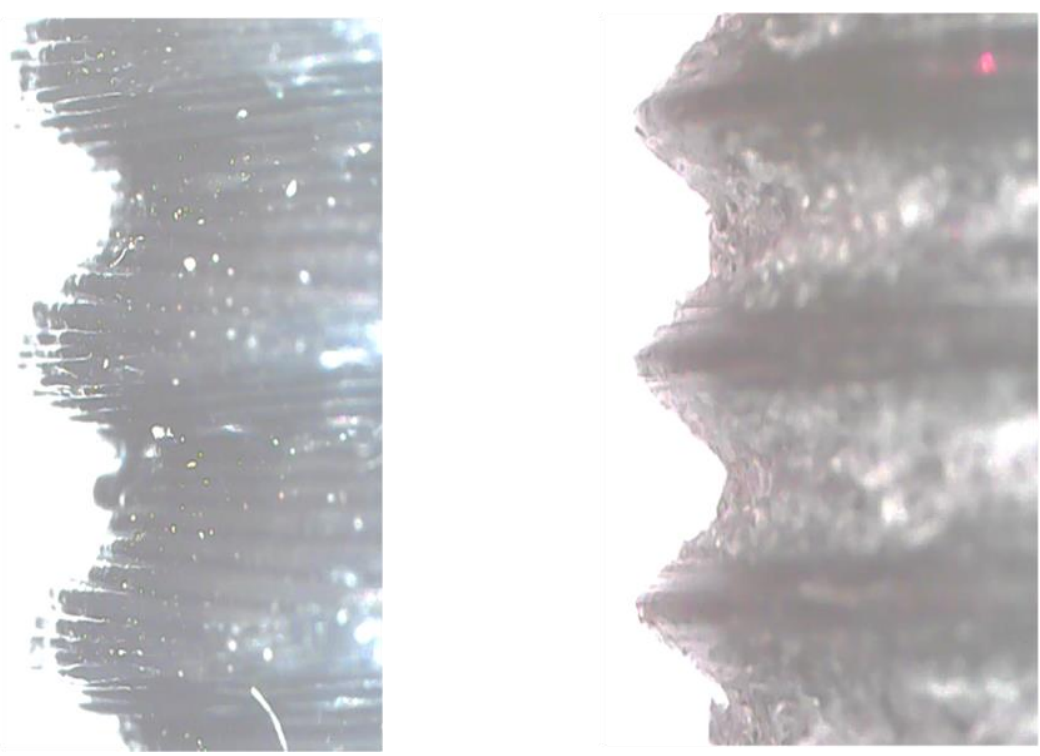

Figure 4: Microscopic images of the thread without (left) and with re-rolling treatment (right)

In order to test the performance of the repair method, a faulty geometry was then produced in the form of a thread flank of an M10x1.5 metric standard thread reduced on one side to the core diameter over a length of approx. $10 \mathrm{~mm}$. Subsequently, another thread repair was performed using the manual rolling method. Figure 5 shows the test result in the comparison before and after. It can be seen that the thread was clearly restored in the area of the designed defect (compare marking in Figure 5).

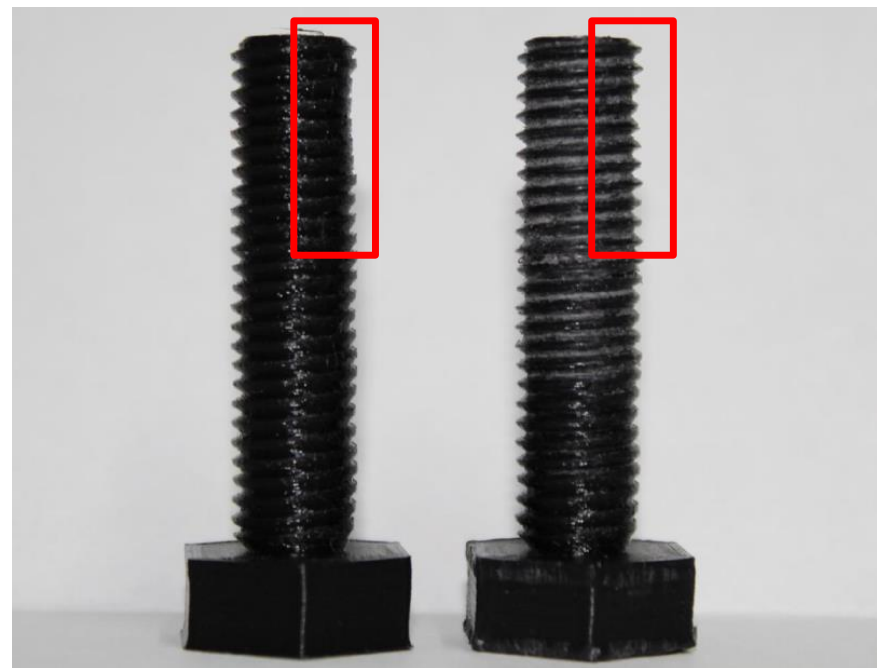

Figure 5: Comparison of pre-damaged specimen (left) and resulting re-rolled thread (right) 
In a further test, the subsequent thread repair in the extreme case of a rough basic shape of a cylindrical shaft with a hexagonal head was investigated. The specimens were printed both in vertical position (layered structure parallel to the head contact surface) and horizontal position (layered structure parallel to the longitudinal axis). Following the work of Jo et al., heat-treated specimens (annealed at $160^{\circ} \mathrm{C}$ for 3 hours) were also examined (Jo et al., 2018). In all cases it was found that the thread rolling process is not suitable for the initial shape embossing in FDM printing. Although both the layer structure parallel to the longitudinal axis and the heat treates specimen significantly raised the endurability of the PLA material against deformation, massive damage to the material in the form of torn edge layers was observed each time the complete geometry was embossed using thread rolling, which made the component unusable.
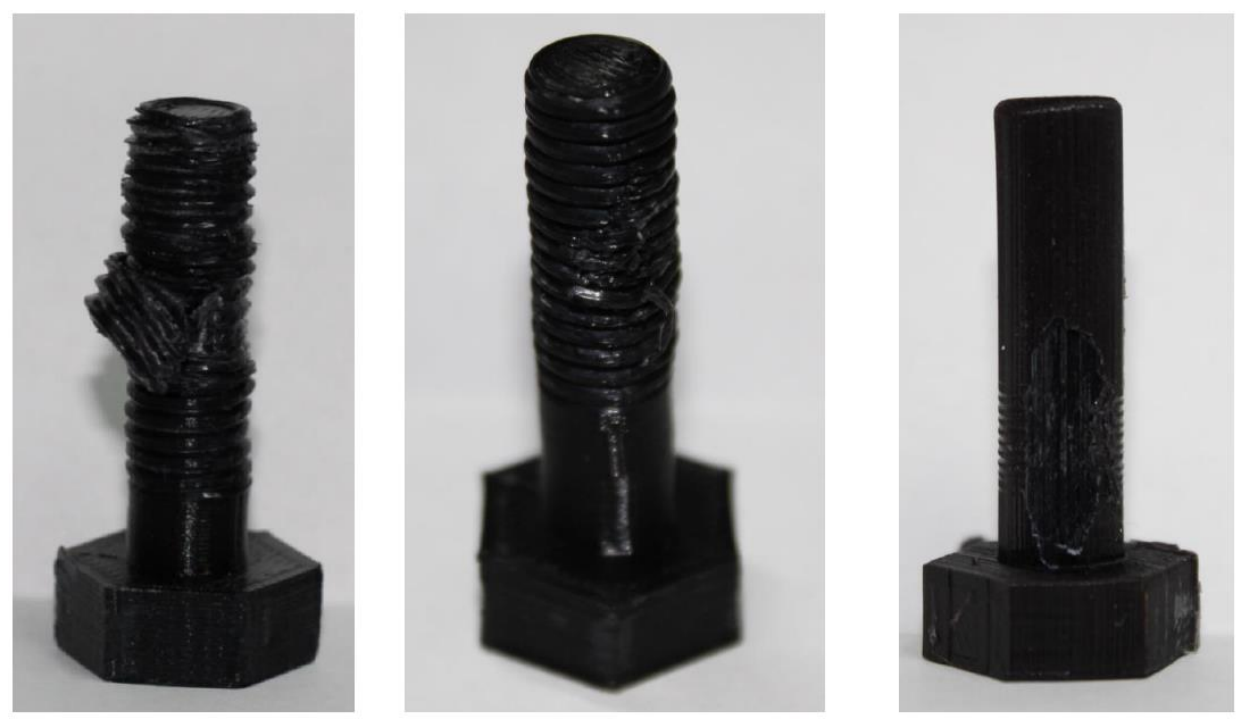

Figure 6: Faulty initial shape imprint with horizontal layers (left), vertical layers (middle) and heat-treated basic geometry (right) all after rolling application

It can be stated that the method of thread repair by means of thread rolls is excellently suited for the repair of failed FDM prints. The loss of material compared to thread cutting is negligible. In all cases of repair, a standard steel nut with a standard thread could be screwed onto the repaired geometry without any problems, which clearly demonstrates the potential of this technology.

In contrast to the repair, the process is unfortunately not suitable for the direct impressing of a standard thread in the context of FDM pressure, since damage occurs through chipping off individual threads from the base body, which is attributed to excessive shear forces in the course of the inspection.

In order to evaluate the load capacity of the repaired threads, a load test in the form of a static linear tensile test was then carried out.

\subsection{Comparison of printing, cutting and rolling}

In an experimental exploration, M8x1.25 threaded screws made of PLA have been examined in regard to their load bearing capacity. In addition to the untreated and iteratively optimized printed product, a reference in terms of a screw post-treated with thread-cutting was also examined using a common die. The rolled threaded screw was printed on the basis of the faulty model from chapter 4.1 and then cold formed using the rolling thread tool.

For comparability, all screws were printed from the same material of the same filament spool with the default settings of an Anycubic i3 Mega desktop printer and the Cura slicing software with a factory default printer profile. This configuration reflects the stock delivery condition of the printer as it is provided to customers. Three test series with three identical sample geometries each were performed repeatively. This means, each test group consists of a printed thread, a cutted thread and a rolled thread with the same nominal metric values.

As FDM printing with PLA is commonly used for statically loaded parts, such as housings with mounting flanges or support structures, a static load analysis has been performed. In the tensile test, the specimens were screwed into threaded steel sleeves and then loaded with a static tensile force. The tensile load was gradually increased by $3 \mathrm{~kg}$ from $45 \mathrm{~kg}$ at the beginning until a maximum of $66 \mathrm{~kg}$. In 
the case of damage, a brittle fracture without significant necking was observed throughout all specimen, see Figure 7.

Despite the different material, the behaviour of the specimens is similar to that of the specimens investigated by Kinzel (Kinzel, 2015). This is astonishing due to the fact that Kinzel's specimen are commercial steel screws, whereas in the present study case polylactide filament (PLA) was used. The very low elongation at break could be due to the material deformation that took place during thread rolling. However, this hypothesis has to be verified in further investigations.
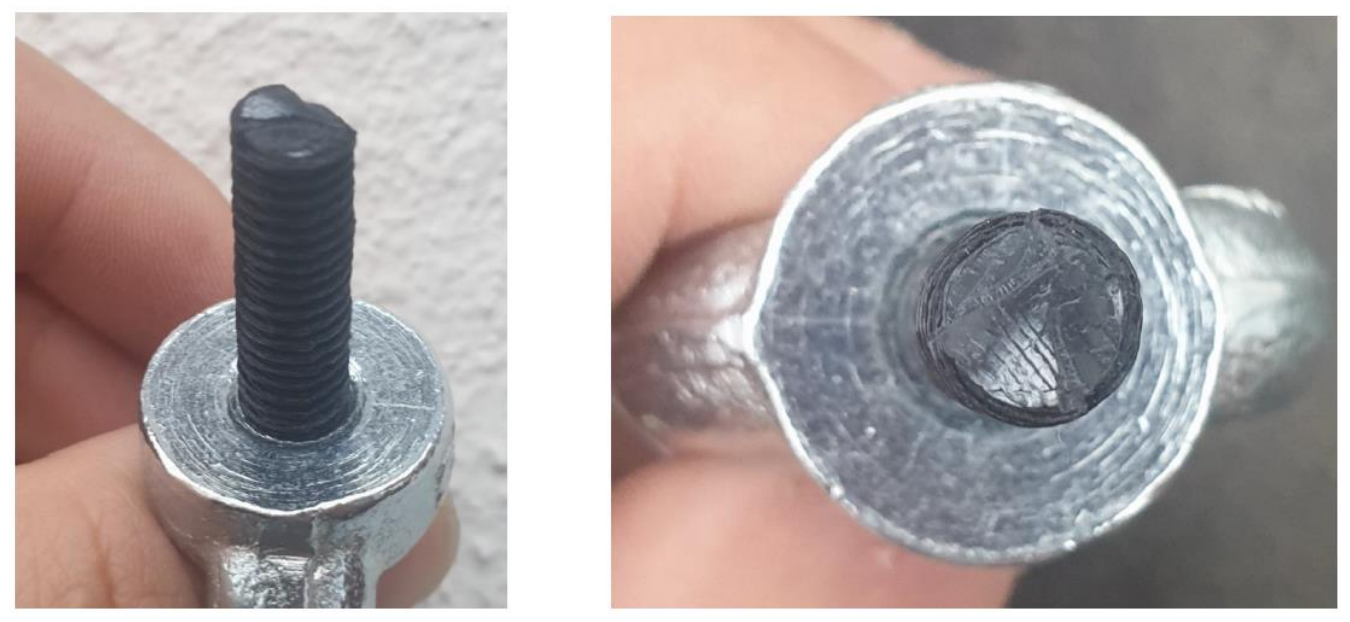

Figure 7: Failure pattern of a tensile specimen

The final evaluation of the tensile test results showed that the thread geometry repaired by thread rolling has the highest tensile strength in two of three test groups.

Table 1: Tensile test results

$\begin{array}{cccc}\begin{array}{c}\text { Resilience } \\ {[\mathrm{kg}]}\end{array} & \text { Test group 1 } & \text { Test group 2 } & \text { Test group 3 } \\ \begin{array}{c}\text { Printed } \\ \text { thread }\end{array} & 45 & 66 & 63 \\ \text { Cutted thread } & >66 & 45 \text { (thread loosen) } & 51 \\ \text { Rolled thread } & 66 & 60 & >66\end{array}$

Finally, it can be assumed that the rolling produces a better overall surface structure than pure FDM printing. In addition, the individual print layers are smeared with each other during the fine rolling process, which creates a more homogeneous structure. In the application case of the embossing of metric standard threads, it can be seen that the after-treatment of the printed products with the presented method results in significantly more pronounced thread flanks and an overall thread profile that conforms closer to the standard. In all cases standard stock nuts could be screwed to the rethreaded specimen without additional effort, which offers potential for the combination of stock parts and $3 \mathrm{~d}$ printing objects without machine specific try-and-error iterations to find the best printing parameters for each thread size

\section{CONCLUSION}

In this paper, a method was demonstrated for solving the problem of quality assurance in the production of standardized threads in the field of FDM 3D printing. As a result, FDM parts can be optimized quickly and cost-effectively with the aid of a new process. Currently, the presented technology is only available for external threads up to a diameter of $110 \mathrm{~mm}$, but a tool development for the repair of internal threads is currently being developed by the company Silbertool GmbH. In contrast to thread cutting, the process does not require significant material removal and enables the application of standard parts to threaded studs of an FDM printed product across all printing devices. 
From the strength investigations, it can be deduced that the maximum load-bearing capacity of a metric external thread made of PLA can be achieved in the FDM process by means of a print with standard parameters and post-processing using the thread rolling method. Regarding the tensile strength of the repaired threaded bolt, cyclic loading has to be investigated in further studies to respect the notch sensivity of the overall geometry. The maximum resilience presented in this study does not contain any safety factors and should not be used for a safety-relevant design of functional components.

The investigations presented build up the basis for further studies at the Institute for Product Innovations at the University of Wuppertal. In future research, the relationship between the repair method and the brittle fracture behavior is to be investigated. Furthermore, larger groups of thread profiles and bolt series are to be tested for suitability regarding the presented method.

\section{ACKNOWLEDGMENTS}

We would like to thank Silbertool GmbH for providing their tools free of charge and with an open mind.

\section{REFERENCES}

Horvath, J. and Cameron, R. (2014), Mastering 3D Printing, Mastering 3D Printing, Apress, Berkeley, available at: https://doi.org/10.1007/978-1-4842-5842-2.

Jo, W., Kwon, O.C. and Moon, M.W. (2018), "Investigation of influence of heat treatment on mechanical strength of FDM printed 3D objects", Rapid Prototyping Journal, Vol. 24 No. 3, available at: https://doi.org/10.1108/RPJ-06-2017-0131.

Kinzel, A. (2015), Research Report 20151540-E, Hannover: Materials Testing Institute for Materials and Production Engineering, available at: https://silbertool.com/wp-content/uploads/2020/04/MPA-TestSilbertool-en.pdf.

Klocke, Fritz (2013): Massive Forming. In: Fritz Klocke (Hg.): Manufacturing Processes 4. Forming. Berlin, Heidelberg: Springer Berlin Heidelberg; Imprint; Springer (RWTHedition), S. 169-291.

Kuznetsov, V.E., Solonin, A.N., Tavitov, A.G., Urzhumtsev, O.D. and Vakulik, A.H. (2019), "Increasing of strength of FDM (FFF) 3D printed parts by influencing on temperature-related parameters of the process", Rapid Prototyping Journal, available at:https://doi.org/10.20944/preprints201803.0102.v2.

Sculpteo. (2020), The State of $3 D$ Printing, Villejuif, France, available at: https://www.sculpteo.com/de/ebooks/state-of-3d-printing-report-2020/.

Silbertool. (2020a), “The Process. Thread saver - full recovery of damaged threads", available at: (accessed 9 December 2020).

Silbertool. (2020b), "Silbertool - The rolling process", available at: https://www.youtube.com/watch?v=eXUgCnIgIpQ (accessed 9 December 2020).

Systems, D. (2020), “A.I.M.S. - autonomous intelligent management system", available at: https://www.kickstarter.com/projects/delcossystems/aims-easily-stop-any-print-failure-withai?ref=discovery\&term=3D printer (accessed 10 December 2020).

Thingiverse. (2020), "Thingiverse overview", available at: https://accounts.thingiverse.com/register (accessed 11 December 2020).

Toor, Ravi (2019): The 3D Printing Waste Problem. Online verfügbar unter https://www.filamentive.com/the3d-printing-waste-problem/, zuletzt aktualisiert am 27.11.2019, zuletzt geprüft am 23.03.2021.

VDI. (1989), VDI 3174 - Rolling of External Threads, Berlin: Beuth Verlag, Germany, p. 14. 